\title{
Correction to: Interaction of opioid with insulin/IGFs signaling in Alzheimer's disease
}

\author{
Sadegh Salarinasab ${ }^{1} \cdot$ Leila Salimi $^{2} \cdot$ Neda Alidadiani $^{3} \cdot$ Elhameh Shokrollahi $^{1} \cdot$ Pishva Arzhanga $^{4}$. \\ Saedeh Karbasforush ${ }^{1} \cdot$ Faroogh Marofi $^{5} \cdot$ Mahdieh Nasirzadeh $^{6} \cdot$ Reza Rahbarghazi $^{2,7,8}$ (D) Alireza Nourazarian ${ }^{1,9}$. \\ Masoud Nikanfar ${ }^{10}$
}

Published online: 18 April 2020

(C) Springer Science+Business Media, LLC, part of Springer Nature 2020

\section{Correction to: Journal of Molecular Neuroscience https://doi.org/10.1007/s12031-020-01478-y}

The original version of this article unfortunately contained mistake in the Author Group section. Reza Rahbarghazi's family name was inadvertently spelled as "Rahbarghzi".

The original article has been corrected.

The online version of the original article can be found at https://doi.org/ 10.1007/s12031-020-01478-y

Reza Rahbarghazi

Rezarahbardvm@gmail.com; rahbarghazir@tbzmed.ac.ir

$\triangle$ Alireza Nourazarian

Nourazariana@tbzmed.ac.ir; alinour65@gmail.com

1 Neuroscience Research Center, Institute of Neuropharmacology, Kerman University of Medical Science, Kerman, Iran

2 Stem Cell Research Center, Tabriz University of Medical Sciences, Tabriz, Iran

3 Department of Cardiac Surgery, Friedrich-Alexander-University Erlangen-Nürnberg, Krankenhausstr. 12, 91054 Erlangen, Germany

4 Department of Biochemistry and Diet Therapy, Faculty of Nutrition and Food Sciences, Tabriz University of Medical Sciences,

Tabriz, Iran
Department of Hematology, Faculty of Medicine, Tabriz University of Medical Sciences, Tabriz, Iran

6 Department of Biochemistry, Faculty of Medicine, Urmia University of Medical Sciences, Urmia, Iran

7 Drug Applied Research Center, Tabriz University of Medical Sciences, Tabriz, Iran

8 Department of Applied Cell Sciences, Faculty of Advanced Medical Sciences, Tabriz University of Medical Sciences, Imam Reza St, Golgasht St, Tabriz 51666-14756, Iran

9 Department of Biochemistry and Clinical Laboratories, Faculty of Medicine, Tabriz University of Medical Sciences, Golgasht St, Tabriz 51666-16471, Iran

10 Department of Neurology, Faculty of Medicine, Tabriz University of Medical Sciences, Tabriz, Iran 